\title{
Minimally invasive surgical method to detect sound processing in the cochlear apex by optical coherence tomography
}

Sripriya Ramamoorthy

Yuan Zhang

Tracy Petrie

Anders Fridberger

Tianying Ren

Ruikang Wang

Steven L. Jacques

Alfred L. Nuttall 


\title{
Minimally invasive surgical method to detect sound processing in the cochlear apex by optical coherence tomography
}

\author{
Sripriya Ramamoorthy, ${ }^{a}$ Yuan Zhang, ${ }^{a}$ Tracy Petrie, ${ }^{b}$ Anders Fridberger, ${ }^{c}$ Tianying Ren, ${ }^{a}$ Ruikang Wang, ${ }^{d}$ \\ Steven L. Jacques, ${ }^{\mathrm{b}, \mathrm{e}}$ and Alfred L. Nuttall ${ }^{\mathrm{a}, \mathrm{f}, \star}$ \\ a Oregon Health and Science University, Oregon Hearing Research Center, Department of Otolaryngology, 3181 SW Sam Jackson Park Road, \\ NRC 04, Portland, Oregon 97239, United States \\ bOregon Health and Science University, Department of Biomedical Engineering, 3303 Bond Street, Portland, Oregon 97239, United States \\ 'Linköping University, Department of Clinical and Experimental Medicine, Cell Biology SE-58185, Linköping, Sweden \\ dUniversity of Washington, Department of Bioengineering, William H. Foege Building, P.O. Box 355061, 3720 15th Avenue NE, Seattle, \\ Washington 98195-5061, United States \\ ${ }^{e}$ Oregon Health and Science University, Department of Dermatology, Portland, Oregon 97239, United States \\ fUniversity of Michigan, Kresge Hearing Research Center, Ann Arbor, Michigan 48105, United States
}

\begin{abstract}
Sound processing in the inner ear involves separation of the constituent frequencies along the length of the cochlea. Frequencies relevant to human speech $(100$ to $500 \mathrm{~Hz})$ are processed in the apex region. Among mammals, the guinea pig cochlear apex processes similar frequencies and is thus relevant for the study of speech processing in the cochlea. However, the requirement for extensive surgery has challenged the optical accessibility of this area to investigate cochlear processing of signals without significant intrusion. A simple method is developed to provide optical access to the guinea pig cochlear apex in two directions with minimal surgery. Furthermore, all prior vibration measurements in the guinea pig apex involved opening an observation hole in the otic capsule, which has been questioned on the basis of the resulting changes to cochlear hydrodynamics. Here, this limitation is overcome by measuring the vibrations through the unopened otic capsule using phase-sensitive Fourier domain optical coherence tomography. The optically and surgically advanced method described here lays the foundation to perform minimally invasive investigation of speech-related signal processing in the cochlea. ๑ The Authors. Published by SPIE under a Creative Commons Attribution 3.0 Unported License. Distribution or reproduction of this work in whole or in part requires full attribution of the original publication, including its DOI. [DOI: 10.1117/1.JBO.21.2.025003]
\end{abstract}

Keywords: optical coherence tomography; optical interferometry; phase-sensitive Fourier domain optical coherence tomography; cochlear vibration; speech; probe.

Paper 150443PR received Jun. 30, 2015; accepted for publication Dec. 23, 2015; published online Feb. 2, 2016.

\section{Introduction}

Sound entering the ear stimulates the cochlea and launches a fluid-structure traveling wave along the structures in the inner ear. The constituent sound frequencies are separated along the length of the cochlea with high frequencies near the base, which is closer to the middle ear, and low frequencies near the apex. The sound-induced vibrations inside the cochlea are highly complex in a living mammal and are vulnerable to damage. These vibrations could, therefore, provide important information on the mechanisms of normal and impaired hearing. Most previous measurements of inner ear vibrations were limited to the first surface in the optical view, which is the basilar membrane in the basal turn and the reticular lamina or the tectorial membrane in the apical turn, upon opening the bony otic capsule. ${ }^{1}$ Furthermore, reflective beads were introduced to enhance the signal-to-noise ratio, as inner ear tissues have poor reflectivity.

Phase-sensitive Fourier domain optical coherence tomography $(\mathrm{PhS}-\mathrm{FDOCT})$ is ideal to measure inner ear vibrations because it can detect vibrations of samples with very low native reflectance without the need to introduce reflective objects. Vibrations on the order of a fraction of a nanometer can be

*Address all correspondence to: Alfred L. Nuttall, E-mail: nuttall@ ohsu.edu measured across tissue and over a wide frequency range. Additionally, it provides the capability to simultaneously image the structure to identify where the vibrations are detected. This technique has recently been used to measure inner ear vibrations without opening the cochlea in the hook region of the guinea pig cochlea, ${ }^{2}$ hook and next turn in the mouse cochlea, ${ }^{3}$ and in the mouse apical turn. ${ }^{4}$

The fundamental frequency of the human voice ranges from 100 to $300 \mathrm{~Hz}$, and a large part of the frequency range used in music has fundamental frequencies and at least some harmonics at frequencies below $1000 \mathrm{~Hz}$. The ability to hear a low stimulus frequency is therefore fundamental for speech and music perception, and, indeed, oral communication becomes difficult if low-frequency hearing is disturbed. It is believed that the cochlear processing of low frequencies in the apex is different from the processing of high frequencies in the basal region. ${ }^{1}$ The different behavior in the apex could be concomitant with the fact that the auditory nerve phase-locks to sound stimulus at low frequencies (below $\sim 4000 \mathrm{~Hz}$ ), whereas it is not phase-locked at higher frequencies.

While much information from physiologically intact preparations is available from the high-frequency or basal regions of the cochlea, ${ }^{1,5,6}$ this is not the case for the apical regions. One problem is that previous techniques required opening the cochlea, which is doable at high frequencies (because acoustic 
wavelengths are smaller than the cochlear dimensions), but the response of the apical turn may be altered by such observation windows in the bone (e.g., Ref. 7). The problem is compounded by the anatomical inaccessibility of the guinea pig cochlear apex, which has meant that previous studies were either performed in vitro, or in preparations where an extensive and potentially damaging surgical approach was necessary. The history of cochlear imaging and interferometry is discussed in detail in Ref. 8.

We have developed measurement techniques and surgical approaches that improve this situation. The method described here enables us, for the first time, to measure the cochlear mechanical response to frequencies $<1000 \mathrm{~Hz}$ in the absence of artificial reflectors or observation windows through the bone. The method is based on two technical advancements: (1) optical, to allow optical penetration into the cochlea, in particular to the organ of Corti and tectorial membrane, without opening the otic capsule and (2) anatomical, to allow measurements apically along the cochlear axial direction with reduced surgery compared with that used thus far. The optical development is based on PhS-FDOCT and the anatomical development on the novel idea of using a mirror to direct the beam axially, thus eliminating the need for ventral surgery. Since the current surgery is less traumatic and the fluid boundary conditions are unaltered, these two developments together enable gathering of important, less artifact-prone vibration data for understanding sound processing in the low-frequency region of the cochlea.

\section{Materials and Methods}

\subsection{Ethical Approval}

Measurements reported in this article were made in vivo in normal-hearing albino guinea pigs of either sex (250 to $350 \mathrm{~g}$ ). Albino animals are expected to have the same normal cochlear mechanics as pigmented, and the lack of pigmentation reduces light scatter in the cochlea. All procedures in this study were approved by the Institutional Animal Care and Use Committee of Oregon Health and Science University.

\subsection{Experimental Setup}

The schematic of the PhS-FDOCT system setup used in this study is shown in Fig. 1. The optical source is a broadband superluminescent diode (SLD, SuperLum) with a central wavelength of $840 \mathrm{~nm}$ and a spectral bandwidth of $45 \mathrm{~nm}$. The light from the SLD is coupled into a fiber-based Michelson interferometer via an optical circulator with a coupling ratio of 90:10. In the reference arm, the light is delivered onto a stationary mirror. The light in the sample arm is focused onto the sample via an objective lens with a focal length of $30 \mathrm{~mm}$. The sample is scanned along the $x$-direction by a galvo mirror (GVSM002, Thor Labs) to acquire reflectance image using B-scan or to acquire vibration at multiple spatial points using M-B mode scanning.

A $2 \times 2$ optical fiber coupler recombines the light backscattered from the sample and reflected from the reference mirror. This combined beam is routed to a purpose-built, high-speed spectrometer via the optical circulator. The spectrometer consists of a 30-mm focal length collimator, a 1200-lines/mm transmission grating, an achromatic lens with $100 \mathrm{~mm}$ focal length, and a 12-bit, 4096-pixel CMOS line-scan camera (Basler Sprint spL4096-140 km), of which only 2048 pixels are used, to reduce

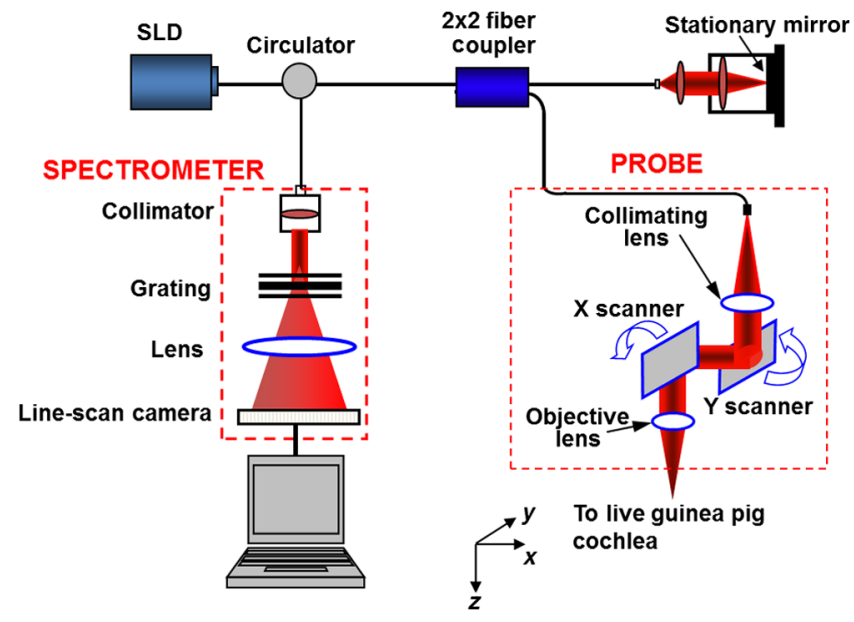

Fig. 1 Schematic of the PhS-FDOCT system used in this study to measure in vivo vibrations in guinea pig cochlear apex through the otic capsule. SLD with center wavelength of $840 \mathrm{~nm}$.

data-acquisition time. To improve the signal-to-noise ratio, we use vertical binning as well as horizontal binning, both over 2 pixels, which effectively yields 1024 pixels. The maximum line-scan rate of the camera is $140 \mathrm{kHz}$. We use a reduced sample rate of $10 \mathrm{kHz}$ to improve the signal-to-noise ratio, as the maximum frequency of interest is $<1 \mathrm{kHz}$ in this study.

The coherence length and central wavelength of the light source determine the axial resolution, which, in the current instrument, is $\sim 5 \mu \mathrm{m}$ in tissue, an advantage of the $840-\mathrm{nm}$ source. The axial resolution is calculated using the formula

$l_{c}=\frac{4 \ln (2)}{\pi} \frac{\lambda_{0}^{2}}{n \Delta \lambda}=6 \mu \mathrm{m}$

in air and 4.3 in tissue for $n=1.4 .{ }^{9}$ In PhS-FDOCT, the entire penetration depth of the beam is used at once to image along the optical axis of the system. To generate images of the cochlear structures, a computer-controlled galvo mirror moves the beam across the preparation. Once the organ of Corti is identified, the phase and amplitude of sound-evoked motion is determined for every image pixel. Due to the large penetration depth of infrared light, the technique makes it possible to image and measure the vibration of all cellular and noncellular structures of the organ of Corti in the apical turn. The good penetration of the beam makes opening of the cochlea unnecessary. Vibrations are measured over a wide frequency range, and structures are simultaneously imaged, which allows us to determine the sound-evoked vibration of all cells within the organ of Corti, not only those at the surface.

\subsection{Surgical Preparation}

Young guinea pigs weighing 250 to $350 \mathrm{~g}$ were anesthetized by an intraperitoneal injection of a combination of ketamine $(40 \mathrm{mg} / \mathrm{kg})$ and xylazine $(10 \mathrm{mg} / \mathrm{kg})$. Supplemental $1 / 2$ doses were given every hour or when need was indicated by paw withdrawal to toe pinch. The guinea pig was mounted on a heated head holder. A tracheotomy and placement of a short section of ventilation tubing allowed free breathing. No artificial respiration was needed, and motion from respiration or cardiovascular vibrations did not significantly affect the measurement noise floor. Surgical operation was performed to expose the left 
bulla, which was then incised to open for access to the cochlea. ${ }^{10}$ The surgical dissection to expose the auditory bulla was from a ventral direction, and the bulla was widely opened to expose the cochlea but preserve the tympanic membrane. With a widely opened bulla, the apex of the cochlea becomes visible. During the surgery as well as during the experiment, the core temperature of the animal was maintained at 37 to $38{ }^{\circ} \mathrm{C}$ by a heating blanket and a rectal thermometer, controlled by a servo temperature controller (FHC, Bowdoinham, Maine).

\subsection{Measurement in Axial and Radial Directions}

For apex vibration measurements in the axial direction, it is not possible to simply adjust the head orientation in a guinea pig. The reason is evident from the location of the bulla and the cochlea inside the skull shown in Fig. 2. Because the axial viewing direction is occluded by the jaw and associated neck tissue, extensive surgery is needed to remove part of the jaw and musculature to expose the apex in the axial direction. However, such extensive surgery takes significant time and adds physiological stress and blood loss, which can affect the viability of the guinea pig for long-duration studies while under anesthesia. In addition, the hearing sensitivity could be affected by extensive surgery, and we lack easy-to-use and reliable electrophysiological indicators of cochlear function at these low frequencies (unlike compound action potential and cochlear microphonics at high frequencies for basal turn studies $;^{10}$ see also Ref. 11).

To overcome this issue and gain access to the axial direction, we use a mirror device that has two right-angled faces with large openings and a reflective front surface mirror is attached to a 45-deg slanted face [Fig. 3(c)]. This device is placed inside the live animal's bulla facing the cochlea, as shown in

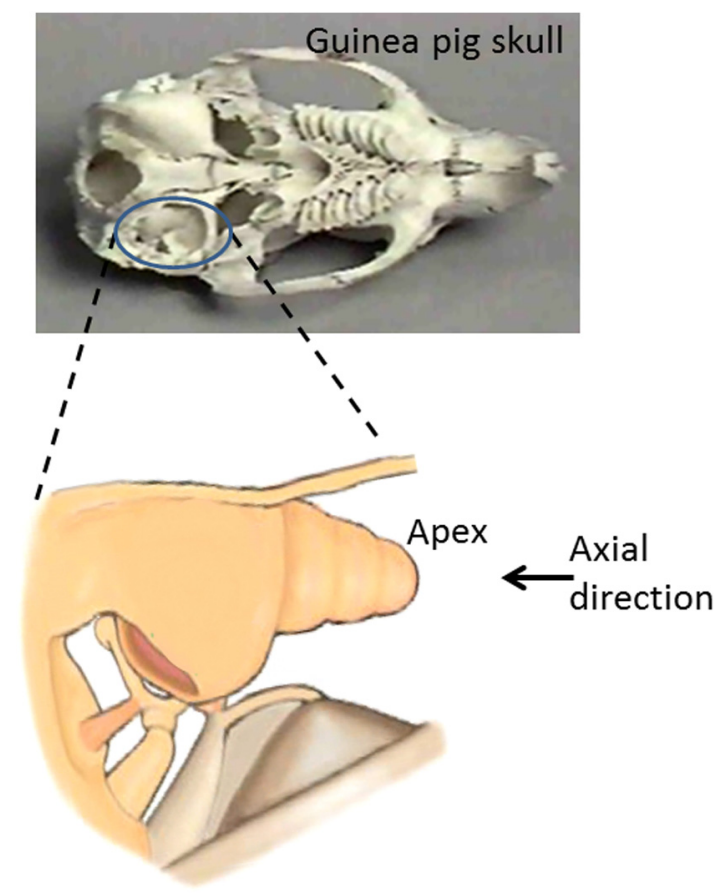

Fig. 2 The guinea pig skull (top) shows the location of the cochlea, and the expanded schematic (bottom) shows the orientation of the apex. Direct optical access to the cochlear apex in the axial direction required in earlier experiments that much of the jaw and neck tissues be removed. Such extensive surgery involved significant blood loss and posed high risk for loss in hearing sensitivity.
Fig. 3(d) and schematically in Fig. 3(a). Because the light is deflected by 90 deg inside the bulla, surgical removal of the jaw and neck tissue is not necessary. This makes it possible to measure vibration using a standard surgical approach developed for the basal turn, a very significant advantage, since extensive dissection is avoided. The placement of the mirror device inside the bulla needs to be done carefully in order to get correct orientation to access the tissues in the cochlear apex. We placed the device inside the bulla by viewing under a dissection microscope and oriented the device such that the top plastic surface was horizontal, as shown in Fig. 3(a).

To measure the vibrations in the radial direction, the animal's head orientation was adjusted and the light from the sample arm was directed at the apex as shown in Fig. 3(b) without the use of the mirror device. The depth of the inner ear structures to be viewed in this direction is larger compared to the depth in the axial direction. Therefore, the otic capsule is thinned, but not opened, in older animals to improve the signal-to-noise ratio. In younger animals (less than five weeks of age), the native bone is already sufficiently thin even for the radial vibration measurement.

\subsection{Sound Stimulation and Data Acquisition}

All sound stimuli were delivered to the ear through a custom speculum fitted tightly into the ear canal to form a closed sound field. An earbud speaker was used to generate the sound. The pressure at the ear canal was precalibrated using a microphone (Bruel and Kjaer type 4138). The data acquisition was done using custom software codes written in Lab View software (National Instruments), which generates the voltage signal to drive the speaker as well as recording the data acquired by the line-scan camera using a camera link. The input voltage to the speaker was adjusted to deliver a constant sound level to the ear. The data recorded by the camera were a function of the optical wavenumber and contained information about the reflectance and vibration of the sample. The postprocessing method described in Ref. 12 was used to derive vibration and reflectance image from the recorded data. The lowest detectable vibration depends on several factors such as the reflectivity of the tissue, animal-to-animal variability, camera sampling rate used, number of averages, etc. For the parameters used in this study (100 to $500 \mathrm{~Hz}, 40$ averages, $10-\mathrm{kHz}$ camera sampling rate, or $4 \mathrm{~s}$ of averaging time), and for the guinea pig apex organ of Corti through the otic capsule, the lowest detectable vibration amplitudes were around $1 \mathrm{~nm}$. Only displacements with signal higher than the mean noise floor by at least five standard deviations were considered for analysis.

The displacement amplitude is given by $A_{\mathrm{D}}=\left(\lambda_{0} \Phi\right) /(4 \pi n)$, where $A_{\mathrm{D}}$ is the displacement amplitude, $\lambda_{0}$ is the center wavelength of the light source, $\Phi$ is the phase of the depth-resolved optical signal, and $n$ is the sample refractive index. The largest displacement that can be measured without phase wrapping is equal to a quarter wavelength of the optical source for $n=1$. In order to reduce optical noise, as well as to improve this maximum displacement limit, velocity is derived via time differentiation of the raw phase data as discussed in Ref. 12. The time differentiation is done numerically by deriving the phase difference between adjacent A-scans. The velocity magnitude is then given by $A_{\mathrm{V}}=\lambda_{0} S /(4 \pi n) * \Delta \Phi$, where $S$ is the camera sampling rate. The maximum velocity measurable without phase wrapping is therefore given by $A_{\mathrm{V}}^{\max }=\frac{\lambda_{o} S}{4 n}$. 
(a) Axial direction

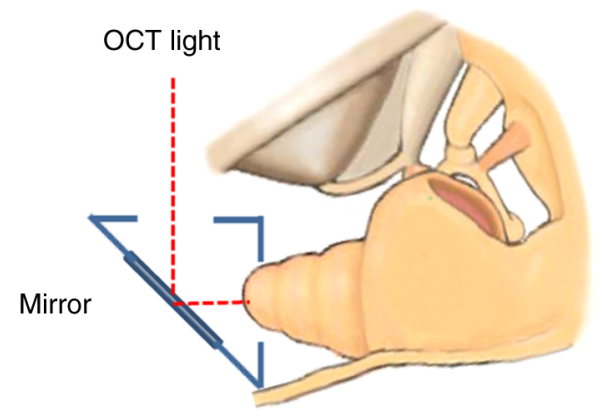

(b) Radial direction

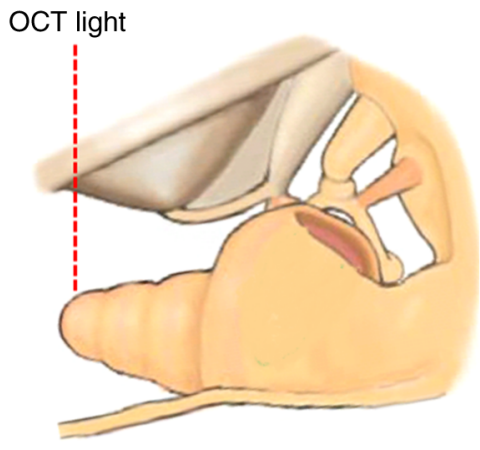

(c) Mirror device

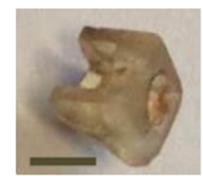

(d) Mirror device inside the bulla

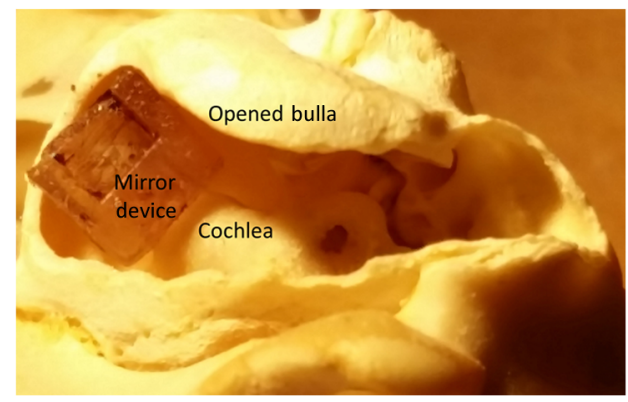

Fig. 3 The method used to measure axial and radial vibrations of the guinea pig cochlear apex. (a) To measure the vibrations in the axial direction, the OCT light from the sample arm is deflected by 90 deg using a mirror device with a reflective front surface mirror placed inside the bulla of the live guinea pig. This step significantly reduces the surgery needed to measure the axial vibrations in the guinea pig apex. (b) The vibrations in the radial direction are measured by direct placement of the animal with the appropriate head orientation in the sample arm, without the use of the mirror device. (c) The mirror device is created by attaching a mirror to the 45-deg surface of a three-dimensional printed prism-shaped plastic part. In this image, the mirror is located on the bottom side. Scale bar $2.5 \mathrm{~mm}$. (d) Mirror device placed inside the bulla in a guinea pig skull for axial direction measurements.

For the detector, we used the Basler Sprint spL4096-140 km CMOS line-scan camera with a maximum sampling rate of $140 \mathrm{kHz}$. However, because this study was done at low frequencies $<1 \mathrm{kHz}$, we operated the camera at a reduced rate of $10 \mathrm{kHz}$ to take advantage of the larger signal-to-noise ratio. Reducing the camera rate further would limit the maximum vibration that can be measured without phase wrapping. In this study, $\lambda_{0}=$ $840 \mathrm{~nm}$. This gives a maximum velocity limit of $0.21,2.1$, and $21 \mathrm{~mm} / \mathrm{s}$ for camera sampling rates of 1,10 , and $100 \mathrm{kHz}$, respectively. Figure 4 shows the corresponding maximum
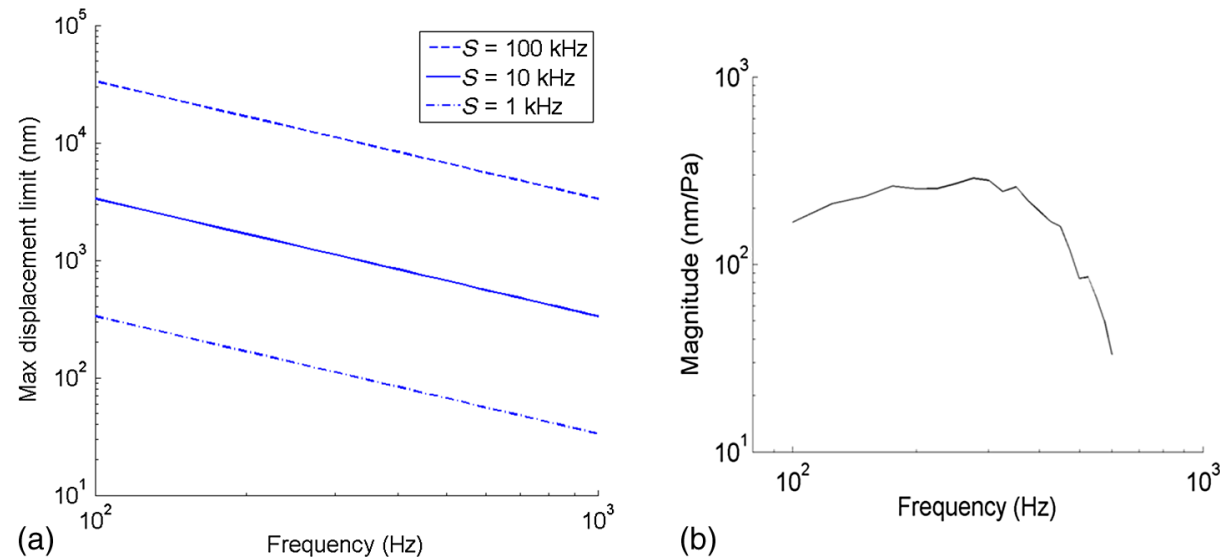

(b)

Fig. 4 (a) Maximum displacement that can be measured using PhS-FDOCT with center wavelength of $840 \mathrm{~nm}$ and for camera sampling rates $S$ indicated in the legend. Note that a quarter wavelength of the optical source is $210 \mathrm{~nm}$. The sound-induced displacements in the guinea pig apex can approach several hundred nanometers for $90 \mathrm{~dB}$ SPL stimulus level, as shown in (b). In the sample axial frequency response shown in (b), the maximum displacement at $94 \mathrm{~dB}$ SPL (or 1 Pascal) is $300 \mathrm{~nm}$ at $300 \mathrm{~Hz}$. This value can be higher in some animals. 
displacement limit versus stimulus frequency for the three camera sampling rates. For the $90 \mathrm{~dB}$ SPL sound stimulus used in this study, the vibrations in the guinea pig apex can be as large as 250 to $500 \mathrm{~nm}$ below $300 \mathrm{~Hz}$ and lower at higher frequencies, as shown by a typical recording in Fig. 4(b). Therefore, we chose to use a $10 \mathrm{kHz}$ camera sampling rate in this study.

\section{Results and Discussion}

\subsection{Vibrations in the Axial Direction}

As described in Sec. 2, a mirror device placed inside the live guinea pig bulla is used to measure the inner ear vibrations in the guinea pig apex through the otic capsule in the axial direction. The reflectance (structural) image is shown in Fig. 5(a), and a schematic is shown in Fig. 5(c) for comparison. The Reissner's membrane, basilar membrane, and the organ of Corti can be identified in the reflectance image. The tectorial membrane appears faint or sometimes not easy to discern. This could be improved by thinning the otic capsule. The axial displacement response in nanometers at $90 \mathrm{~dB}$ SPL, $200 \mathrm{~Hz}$ is shown in Fig. 5(b). The displacement is higher in the organ of Corti compared to the displacement at the basilar membrane. The Reissner's membrane also has a high displacement response. The response pattern has important physiological implications and is indicative of the status of the inner ear.

For the reflectance image shown in Fig. 5(a), 1000 points are used across the radial direction with 200 time averages. For the vibration pattern shown in Fig. 5(b), 25 points are used across the radial direction with 40 time averages. A smaller number of lateral points is used for the vibration recording to save recording time.

\subsection{Vibrations in the Radial Direction}

The vibrations in the radial direction are measured without using the mirror device by positioning the animal's head as shown in Fig. 3(b). Because the depth of the inner ear structures to be viewed in this direction is larger, the otic capsule may need to be thinned (but not opened) in older animals to improve the signal-to-noise ratio. For the data shown in Fig. 6, thinning the (a) Reflectance image

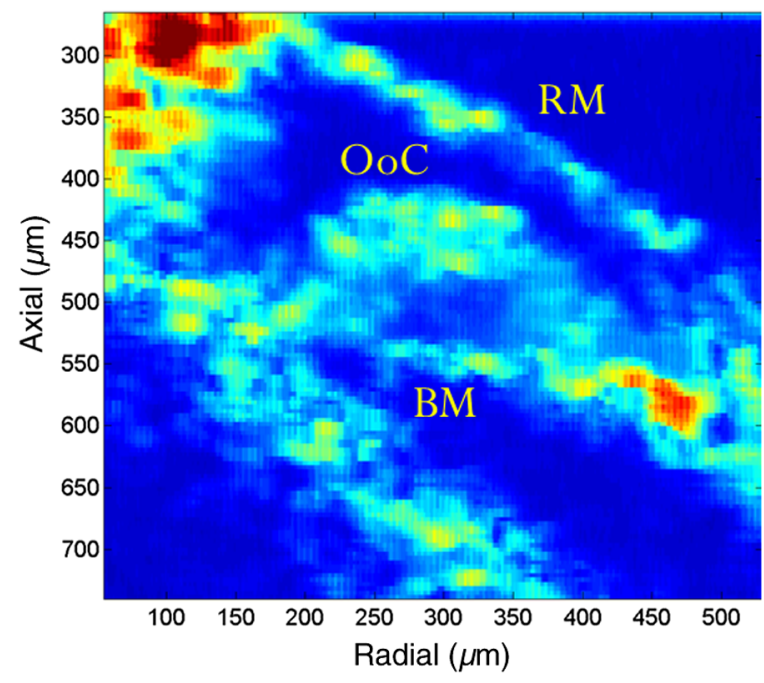

(b) Vibration pattern

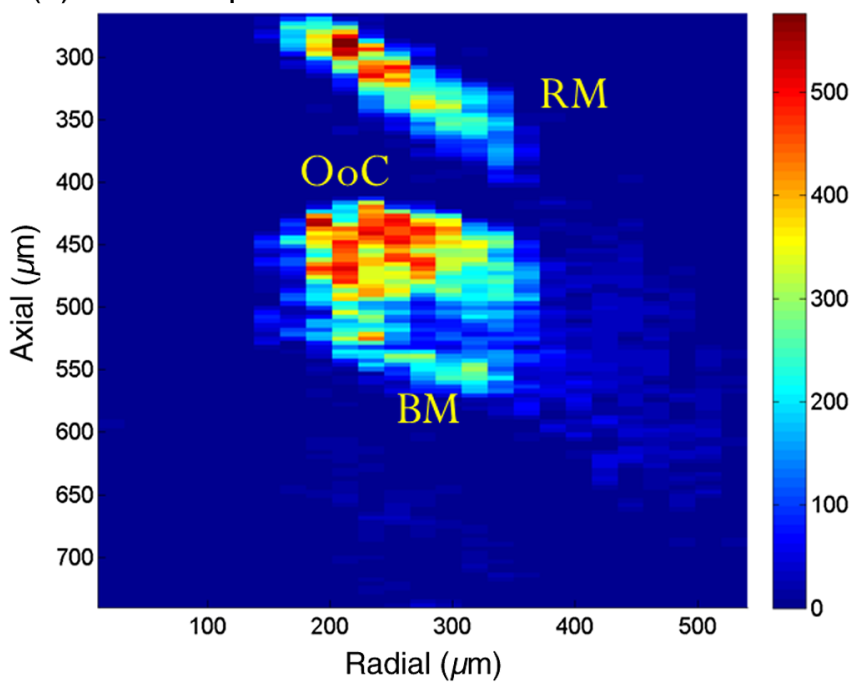

(c) Schematic

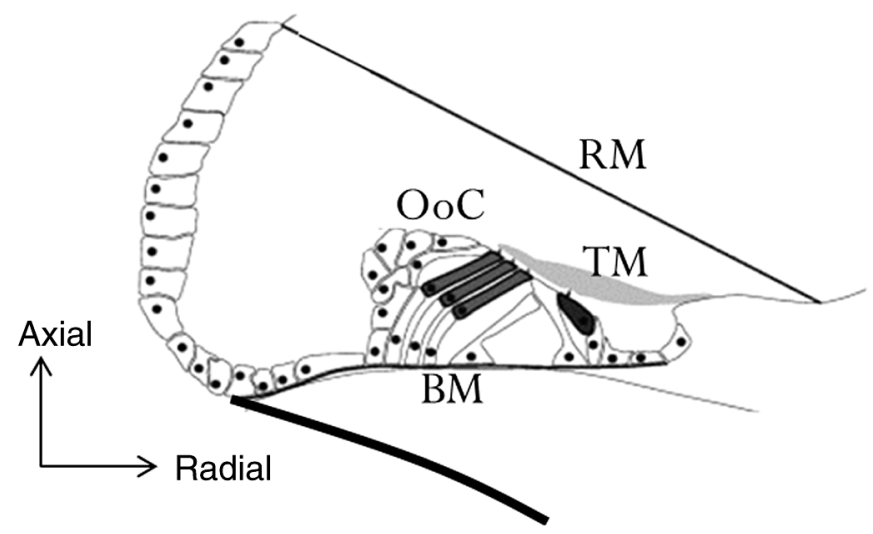

Fig. 5 PhS-FDOCT measurement of the guinea pig apex in the axial direction. (a) Reflectance or structural image of the apex turn viewed in the axial direction using the method described in Fig. 3(a). Otic capsule (not shown) is at the top of the image. A schematic is shown in (c) for comparison (schematic image from web, courtesy of Britta Flock). Axial and radial directions are marked in the schematic. RM, Reissner's membrane; TM, tectorial membrane; BM, basilar membrane; OoC, organ of Corti. (b) Displacement in nanometers in the axial direction for a $90 \mathrm{~dB}$ SPL sound stimulus at $200 \mathrm{~Hz}$. 
(a) Reflectance image

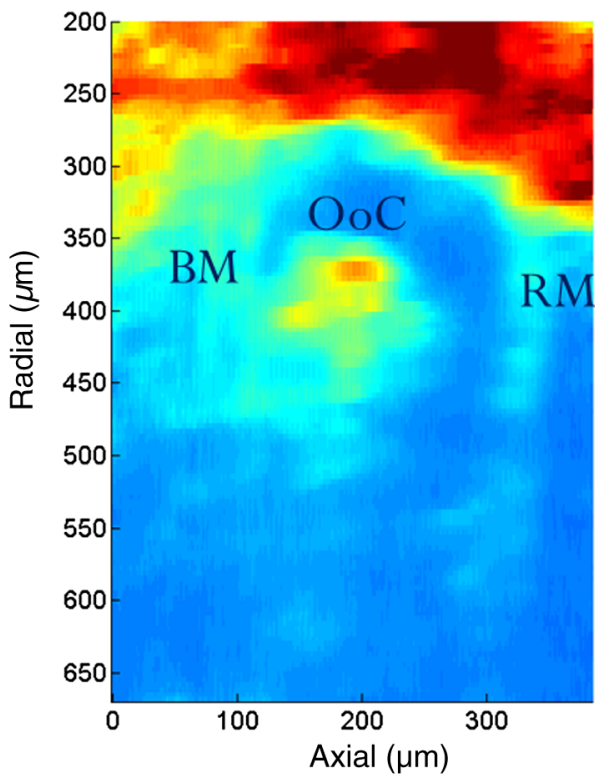

(b) Vibration pattern

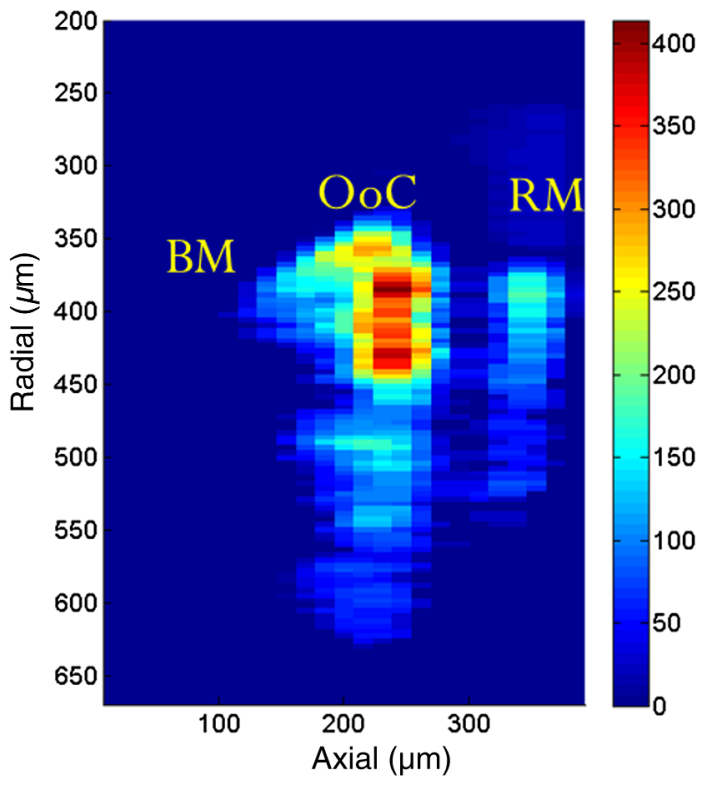

(c) Schematic

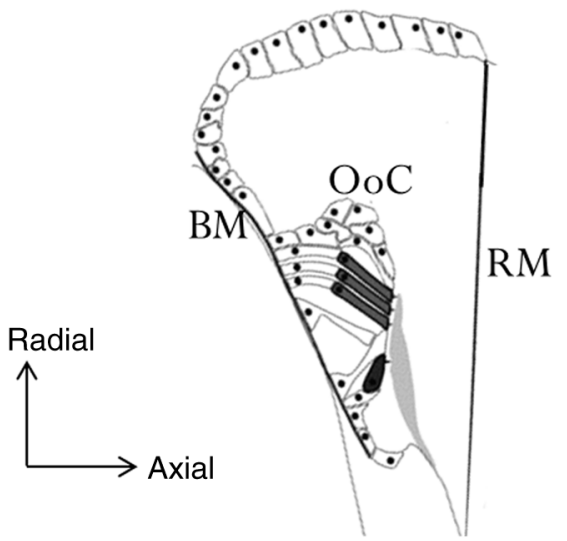

Fig. 6 PhS-FDOCT measurement of the guinea pig apex in the radial direction. (a) Reflectance or structural image of the apex turn viewed in the radial direction using the method described in Fig. 3(b). The schematic is shown in (c) (schematic image from web, courtesy of Britta Flock). RM, Reissner's membrane; BM, basilar membrane; OoC, organ of Corti. (b) Displacement in nanometers in the radial direction for sound stimulus at $150 \mathrm{~Hz}, 90 \mathrm{~dB}$ SPL.

bone was not necessary. The reflectance (structural) image viewed through the bone in the radial direction is shown in Fig. 6(a), and the schematic oriented similarly is shown in Fig. 6(c). Although the deeper structures appear faint, their vibrations can be detected above noise. The radial displacement in nanometers for a $90 \mathrm{~dB} \mathrm{SPL}, 150 \mathrm{~Hz}$ stimulus is shown in Fig. 6(b). These data are from a guinea pig different from that shown in Fig. 5. The radial vibration pattern also contains information on the inner ear physiology.

\section{Conclusions and Future Directions}

This paper describes the method we have developed to measure in vivo vibrations in the apex of the guinea pig cochlea through the otic capsule in two directions (axial and radial) using $\mathrm{PhS}$ FDOCT. A mirror device is placed inside the cochlear bulla to significantly reduce the surgery needed to access the cochlear apex in the axial direction. This method has enabled, for the first time, the measurement of cochlear response to frequencies
$<1000 \mathrm{~Hz}$ in the absence of artificial reflectors or observation windows through the otic capsule. Although the primary soundinduced excitation for the cochlear structures is in the axial direction, it has been hypothesized ${ }^{13}$ that the tectorial membrane resonates radially in response to sound. Models have shown that this resonance could play an important role in active feedback amplification in the cochlea. ${ }^{14-16}$ The tectorial membrane resonance hypothesis has been supported by in vitro experiments in the apex, ${ }^{17}$ but it has not been tested experimentally in vivo. The method developed in this study will enable us to address this and other questions in unopened cochleae of guinea pigs. The apical turn of the guinea pig cochlea has the best frequencies around 100 to $500 \mathrm{~Hz}$. Investigating this region is important to understand how the cochlea processes speech signals.

\section{Acknowledgments}

This work was supported by the following research grants: NIH NIDCD R01DC000141 (A.L.N.); NIH grants R01DC004554 
(T.R.), R01DC010201 (R.W.), and R01DC011796 (S.L.J./A.L. N.); Swedish Research Council K2014-63X-14061-14-5 (A.F.); and Torsten Söderberg Foundation (A.F.).

\section{References}

1. L. Robles and M. A. Ruggero, "Mechanics of the mammalian cochlea," Physiol. Rev. 81(3), 1305-1352 (2001).

2. S. Ramamoorthy et al., "Measurement of in vivo basal-turn vibrations of the organ of Corti using phase-sensitive Fourier domain optical coherence tomography," Proc. SPIE 8565, 85651V (2013).

3. S. Ramamoorthy et al., "Development of a phase-sensitive Fourier domain optical coherence tomography system to measure mouse organ of Corti vibrations in two cochlear turns," AIP Conf. Proc. 1703, 040011 (2015)

4. S. S. Gao et al., "In vivo vibrometry inside the apex of the mouse cochlea using spectral domain optical coherence tomography," Biomed. Opt. Express 4(2), 230-240 (2013).

5. A. L. Nuttall and D. F. Dolan, "Steady-state sinusoidal velocity responses of the basilar membrane in guinea pig," J. Acoust. Soc. Am. 99(3), 1556-1565 (1996).

6. W. S. Rhode, "Observations of the vibration of the basilar membrane in squirrel monkeys using the Mossbauer technique," J. Acoust. Soc. Am. 49(4), 1218 (1971).

7. N. P. Cooper and W. S. Rhode, "Fast travelling waves, slow travelling waves and their interactions in experimental studies of apical cochlear mechanics," Aud. Neurosci. 82, 207-217 (1996).
8. A. L. Nuttall and A. Fridberger, "Instrumentation for studies of cochlear mechanics: from von Bekesy forward," Hear Res. 293(1-2), 3-11 (2012).

9. P. H. Tomlins and R. Wang, "Theory, developments and applications of optical coherence tomography," J. Phys. D 38(15), 2519 (2005).

10. A. L. Nuttall, D. F. Dolan, and G. Avinash, "Laser Doppler velocimetry of basilar membrane vibration," Hear Res. 51(2), 203-213 (1991).

11. J. Lichtenhan et al., "The auditory nerve overlapped waveform (ANOW) originates in the cochlear apex," J. Assoc. Res. Otolaryngol. 15(3), 395-411 (2014).

12. R. K. Wang and A. L. Nuttall, "Phase-sensitive optical coherence tomography imaging of the tissue motion within the organ of Corti at a subnanometer scale: a preliminary study," J. Biomed. Opt. 15(5), 056005 (2010).

13. J. J. Zwislocki and E. J. Kletsky, "Tectorial membrane: a possible effect on frequency analysis in the cochlea," Science 204(4393), 639-641 (1979).

14. J. B. Allen, "Cochlear micromechanics-a physical model of transduction," J. Acoust. Soc. Am. 68, 1660-1670 (1980).

15. R. Nobili and F. Mammano, "Biophysics of the cochlea. II: stationary nonlinear phenomenology," J. Acoust. Soc. Am. 99(4), 2244-2255 (1996)

16. S. Ramamoorthy, N. Deo, and K. Grosh, "A mechano-electro-acoustical model for the cochlea: response to acoustic stimuli," J. Acoust. Soc. Am. 121(5), 2758-2773 (2007).

17. A. W. Gummer, W. Hemmert, and H. P. Zenner, "Resonant tectorial membrane motion in the inner ear: its crucial role in frequency tuning," Proc. Natl. Acad. Sci. USA 93(16), 8727-8732 (1996)

Biographies for the authors are not available. 